\title{
Health of the nation and osteoporosis
}

\author{
Allan St J Dixon
}

Everyone agrees that it is better to prevent disease than to wait until it happens and then try to cure it, but how often is this practical? The recent government consultative document 'The health of the nation' invited comments on the selection of priority key areas where prevention would be a reasonable possibility. The criteria for selection of a key area were: (a) the burden of unprevented disease is great; (b) opportunities for prevention exist; and (c) practical targets can be set.

Key areas considered in the document included coronary heart disease, stroke, smoking, accidents, HIV and AIDS, exercise and fitness, but nothing in the field of osteoarticular disease. At first sight this seems fair. Rheumatoid arthritis, osteoarthrosis, ankylosing spondylitis: we can treat them, but we cannot prevent them. Back pain might be a contender. It certainly causes a large economic burden on industry and a lot is known about ergonomics and load handling, but it is difficult to see how realistic targets could be set. Osteoporosis, however, does fulfil the criteria. The burden of unprevented disease attributable to osteoporosis is immense, prevention is possible, realistic and measurable targets can be set.

Good bones that should last a lifetime are begun in childhood. Peak bone mass is achieved by 30 . Bone loss related to age begins after that, slow at first, quicker later. In women the climacteric acceleration of bone loss additionally exposes them to osteoporosis, so that by the eighth decade one in four women and one in $\mathbf{4 0}$ men will have sustained a fracture related to osteoporosis. Opportunities for intervention to prevent osteoporosis exist at all ages and in both sexes.

The clinical outcome of osteoporosis is fracture, but the timing of this catastrophe depends on chance and trauma, usually trauma of the sort which would not be expected to break a normal bone. This is analogous to coronary artery atherosclerosis, which can develop slowly with few or no symptoms until a catastrophe occurs-that is, the artery is suddenly blocked by a thrombus. The catastrophe could not have happened without the underlying causes. The point is important because the professional perception of osteoporosis has in the past been hampered by acceptance of the trauma, rather than the bone weakness, as the cause of the fractures. Strategies to prevent falls and other traumata are important but less so than strategies to prevent the bone weakness in the first place.
The burden of osteoporosis on the individual subject

The burden of osteoporosis on the individual subject is probably none until a fracture (bone failure) occurs. Long bone fractures if set properly heal normally and once healed are not painful. Fractures of the spine-that is, crush fractures of the vertebral bodies, heal with irreversible displacement, seen on radiographs as wedged or flattened in shape. The resulting distortion of the spinal anatomy is associated with enduring pain in some subjects and arises from the stresses on the spinal ligaments and apophysial joints and probably also from oedema of nerves because of obstruction to nerve root veins.

When several vertebrae have collapsed loss of truncal height may be as much as $200 \mathrm{~mm}$. This reduces the capacity of the thoracic and abdominal cavities and embarrasses the action of the heart, lungs, stomach, and bladder with difficulties with breathing, hiatus hernia, and incontinence. Hip fractures tend to affect elderly subjects. In contrast, spinal fractures tend to affect younger people. As a result, and because of their adverse effects on the viscera and the enduring pain, they may cause more loss of years of good quality life than do long bone fractures, though clinically less common.

\section{Burden on the nation}

The burden on the nation is mainly due to hip fractures. The working party of the Royal College of Physicians ${ }^{1}$ found that patients with hip fractures occupied $20 \%$ of orthopaedic beds; $80 \%$ were in women aged over 65 ; direct hospital costs were $£ 160 \mathrm{~m}$ at $1987-8$ prices; the incidence of hip fractures rose $254 \%$ between 1954 and 1983 - and was then still rising, though it has shown signs of levelling off since. The cost will hardly be less than $£ 300$ m a year at today's prices. About $20 \%$ of patients with a hip fracture are dead in six months and of the remainder, about $50 \%$ lose independence. The annual cost of hip fractures, when these include the increased need for care by others and the increased morbidity after fracture, is likely now to be around $£ 600 \mathrm{~m}$.

Figures for the economic burden of other long bone fractures are more difficult to obtain as such fractures generally do not entail admission to hospital. But any fracture in an elderly person will cause temporary loss of independence and costs will arise from this and also for the primary care services. 
Estimates of the economic burden caused by spinal crush fractures are also difficult to compute. The disability caused by a spinal fracture varies from nil to complete so this burden cannot be counted in terms of the numbers of people in whom this diagnosis is made. The usual outcome in the more severely affected is a reduction in the ability to work or live independently, with a corresponding increase in the need for help from primary care and specialist hospital services and from caring relatives.

The cost of all fractures related to osteoporosis is today at least $£ 1000 \mathrm{~m}$ a year.

\section{Opportunities for prevention}

Opportunities for prevention include both prevention of bone weakness and minimising the burden of hip fractures by preventing falls.

Strategies for prevention of bone weakness are to maximise the strength and mineral content of the skeleton in the years of growth; to maintain the level of the peak bone mass for as long as possible; to slow the rate of bone loss related to age; and, in women, to reduce or prevent climacteric acceleration of bone loss.

Possible targets are an average increase in skeletal height of 10 to $20 \mathrm{~mm}$ in the years of growth; an average increase in peak bone mass of $5 \%$; an average $50 \%$ decrease in the rate of bone loss related to age; and an average reduction of $50 \%$ in the rate of climacteric acceleration of bone loss.

\section{Can these targets be achieved?}

Children grow taller when they are better fed. One survey of Welsh schoolchildren has shown that about $50 \%$ are taking a diet which is calcium deficient. ${ }^{2}$ A scientifically elegant controlled study in homozygous twins has confirmed the benefit of supplementary calcium in childhood. ${ }^{3}$ The implications of this will include maintaining the law for fortifying white bread flour with calcium, a law which is currently threatened by European Community policy against addition of additives. It will also mean that the reintroduction of a free subsidised school milk policy should be considered, along with more attention to the quality of school meals. Achieving the target will certainly include maintaining sports and physical exercise opportunities and strengthening the measures discouraging childhood smoking and alcohol consumption as the latter are associated with an increased risk of osteoporosis.

In young adult life there will be a need to continue good habits with regard to diet, smoking, and alcohol. There is also a need to continue with regular physical activity and this comes down to provision of facilities, particularly in urban areas, and reducing the hours of work and travel to work so that time for exercise is available. The importance of exercise in young adults was emphasised in a recent study. ${ }^{4}$ At the same time there is a paradoxical effect of over exercise and over training in elite women dancers and athletes, some $30-50 \%$ of whom develop oestrogen deficiency, amenorrhoea, and a predisposition to later osteoporosis. ${ }^{6}$
In bone loss related to age there is now a strong case for increasing the calcium content of deficient diets. A recent prospective controlled study has shown that supplemental calcium is important in women more than six years past the menopause. ${ }^{5}$ The authors of that study concluded that 'Healthy older postmenopausal women with a daily calcium intake of less than $400 \mathrm{mg}$ can significantly reduce bone loss by increasing their calcium intake to $800 \mathrm{mg} /$ day'. As in younger age groups continuing appropriate physical exercise, avoiding smoking, and limiting alcohol help protect the skeleton.

Reducing or preventing the climacteric acceleration of bone loss depends almost entirely on adequate oestrogen replacement, balanced with a progestogen in women who have not had a hysterectomy. This is the single most effective and physiological way of maintaining and strengthening the female skeleton. Bone loss caused by falling oestrogen levels is not reversible by calcium supplements.

The climacteric acceleration of bone loss begins about two years before the last menstrual period and continues after it at an exponentially decreasing rate over the next five to 10 years. A woman in her 70s has a bone mass which reflects three things: $(a)$ the bone mass before the climacteric, $(b)$ the postmenopausal rate of bone loss, and $(c)$ its duration. A low initial bone mass, a fast rate of subsequent loss, and an early menopause all predispose to osteoporotic fractures as she ages. Oestrogens will stop the bone loss at any age, but the more years after the menopause the less will be the effect.

\section{Detection of those at risk}

That one in four women will get a fracture related to osteoporosis means, however, that three in four will not. This demands effectiveness of screening methods to detect those most at risk. Modern dual energy $x$ ray absorptiometers (DEXA) can detect those with low bone mass at the menopause, and biochemical markers of bone breakdown can detect fast rates of bone loss. ${ }^{7}$ Also at risk are those with early or surgical menopause, those with a history of primary or secondary amenorrhoea, those with chronic liver, kidney, and digestive diseases, those receiving corticosteroid treatment, and those with various rare diseases. Thus most who are at risk can now be identified. Screening for the individual is now practical and hormone replacement therapy continued for many years will reduced the relative risk of hip fractures in women to 0.5 or less. ${ }^{8}$

There is less certainty about the necessity for screening whole populations. This is partly due to the fact that a number of studies have confirmed that hormone replacement therapy will also reduce the relative risk of coronary heart disease to 0.5 or less. So a woman thought on screening not to be at risk of osteoporosis might be denied this and other benefits of hormone replacement therapy (reviewed in ref 9). Coronary heart disease is now the biggest single cause of death in postmenopausal women, and oestrogen replacement is probably the single most effective way of preventing it. Law 
and others have also argued that whatever the result of screening at the menopause, and whether or not this leads to courses of oestrogen treatment, bone loss related to age eventually takes over to the point that screening in the $50 \mathrm{~s}$ may fail to identify those who are at risk for a hip fracture in their 80 s because all women will by then be at increased risk. ${ }^{4}$ Not everyone agrees. ${ }^{10}$

Partly also there are doubts about the costs and benefits of a policy for screening the population as a whole. A modern DEXA based osteoporosis screening service costs about $£ 80000$ to set up and $£ 30-40000$ a year to run. A service would be needed in every district hospital. Much cheaper are services based on measurement of ultrasonic transmission through bone to give an index of bone density, which has the advantage of not using ionising radiation. So far this method is only practical for the calcaneum and the knee cap, neither of which is often subject to osteoporotic fractures. It might, however, be used to eliminate subjects with dense bone, in whom more expensive DEXA scanning would be a waste of money. Methods based on focused areas of bone using quantitative computed tomographic imaging are unlikely to be generally used because of cost and radiation exposure.

Where DEXA services are available, about $10 \%$ of subjects referred for a bone scan have some underlying cause of osteoporosis, such as hyperthyroidism, or have a bone disease other than osteoporosis. Careful medical supervision of subjects and results is required if opportunities for other therapeutic intervention are not to be missed. This slows up the whole process. A well organised scanning service might scan 20 subjects a day, but their medical supervision would be difficult to fit in. If subjects awaiting a scan were asked to complete a questionnaire this would probably reduce the numbers needing to be seen by a consultant. Experience has shown that the print out provided by the DEXA machine is not enough. Interpretation in the light of individual subjects is also necessary.

The print out gives an answer in grams of bone mineral per square centimetre and also as a percentage of a population norm and whether the result is within or below one or two standard deviations of that norm. Expertise, however, is needed to interpret these norms, which might have been obtained from a population whose ethnic, nutritional, and occupational background was different from that of the subject.

When oestrogen replacement is stopped the rapid but exponentially reducing rate of bone loss is resumed but of course from a higher starting point and studies have shown that even a few years' exposure to postmenopausal oestrogen supplementation will have some effect in reducing the relative risk of later osteoporotic fracture. ${ }^{8}$ There seems to be no reason, however, why a woman who is comfortable with oestrogen replacement should not continue it for life. There is some evidence that the balance of risks and benefits will increase her expectation of life by about three years, and anecdotal evidence that it will increase the quality of that life. At present the proportion of women at high risk of osteoporosis because of early loss of ovarian function who are offered oestrogen replacement is low, and then only for a short and inadequate time. ${ }^{11}$ The proportion of women who go through the climacteric at a normal age and who are offered oestrogen replacement is also low in the United Kingdom and varies enormously from one practice to another and from one country to another. This, and subsequent compliance, depend on the availability of menopause and well-woman clinics where advice and monitoring can take place.

\section{Osteoporosis in men}

The changes in young and adult lifestyles referred to above will help to reduce the burden of later fractures related to osteoporosis, particularly hip fractures in older men. There remain a number of younger men who have spinal osteoporosis and its complications. Surveys have found that in up to $60 \%$ the osteopenia is secondary to some underlying cause-hypogonadism, alcoholism, and corticosteroid treatment being the most common. The prospects for prevention of male spinal osteoporosis look best for those receiving corticosteroids.

From the point of view of rheumatology, osteoporosis is a complication of many chronic rheumatic and disabling diseases, (though not of osteoarthrosis) and will become more so as treatments for those diseases become more effective in prolonging life. Most of the measures available to prevent osteoporosis apply equally to such patients.

\section{Treatment of established osteoporosis}

Osteoporosis is a treatable condition. It seems banal to write this today, but 10 years ago the consensus was that once osteoporosis had occurred there was nothing you could do about it; it was all part of getting older. Defensive myths were established, such as spinal fractures heal in six weeks and anyone complaining of pain lasting longer than that was doing it for social or psychological reasons. The emphasis on the circumstances of the fall that caused the fracture rather than the underlying bone weakness was part of that attitude. The evidence that many subjects with osteoporosis have made functional recoveries with little or no physical pain belies that attitude. As with other conditions for which rheumatologists provide care, treatment of osteoporosis does not just come out of a bottle of pills.

When the $10 \%$ of women and the $60 \%$ of men who have some underlying cause of their osteoporosis have been detected and treated there remain the $90 \%$ and $40 \%$ respectively for whom it is necessary to choose the most suitable treatment. Several treatments are available, of which the most outstanding is hormone replacement therapy in women.

For women the United Kingdom consensus seems to be that hormone replacement therapy can be offered up to age 65 or 10 years after the menopause. In the United States these figures would be 70 and 15 years respectively, parti- 
cularly if the bone density is less than 1.5 standard deviations below the norm.

Endometrial carcinoma is an uncommon tumour but its incidence is multiplied by five or more in postmenopausal women taking continuous unopposed oestrogens. The calendar packs of oestrogen/progesterone combinations reduce the endometrial carcinoma risk to below normal but entail the return to menstrual bleeding in most and premenstrual symptoms in some women-not always welcome. Continuous combined oestrogen and progestogen in younger postmenopausal women and oestrogenonly preparations in older women in whom endometrial atrophy has been established avoid these effects but cannot be generally recommended. Recently licensed for treatment of postmenopausal symptoms (but not for osteoporosis) is tibolone, a synthetic steroid with oestrogenic, androgenic, and progestogenic activities, which does not cause endometrial hyperplasia and has been shown to prevent loss of bone mass in postmenopausal women. ${ }^{12}$

Most of the work showing that oestrogens can protect women against coronary heart disease was done using conjugated equine oestrogens (Premarin), which establishes a more favourable blood lipid profile. As yet combined oestrogen and progestogen forms of hormone replacement therapy, and tibalone, have not had the long follow up needed to show reduction of the risk of coronary heart disease, though these do share a more favourable lipid profile. A worry about long term hormone replacement therapy in the treatment of established osteoporosis is breast cancer. A number of studies have been done, none of them perfect. Prospective, randomised trials take a long time and balanced allocation of risk factors between treated and placebo groups has not been straightforward. Not only age but age since menopause, parity, premenopausal contraceptive use, and positive family history of breast cancer are variables which must be successfully randomised. At present there is evidence of a small increase in the diagnosis of breast cancer after 10 years of hormone replacement therapy, but no evidence of an increase in mortality. The results may reflect increased ascertainment in treated women, who are more regularly examined.

Naturally occurring progestogens have a bone protecting effect but have an unfavourable action on blood lipids and blood pressure. It is accepted that these effects do not cancel the favourable effects of oestrogen in combination hormone replacement therapy.

A negative association between onset of rheumatoid arthritis and previous use of noncontraceptive hormones has been shown in a case/control study. ${ }^{13}$

The liver metabolises oral oestrogens and reduces their effects, in particular by converting oestradiol into oestriol and oestrone. Chemical modification can protect against this but introduces other complications and uncertainties. Transdermal oestradiol is, however, effective as only a small part will go into the hepatic circulation. Administration by medicated adhesive plaster, by inunction, or by implant are all more 'bioactive' than the same amount given by mouth and, incidentally, have caused greater improvement in bone mass.

\section{Treatment other than oestrogen}

Calcitonins, a bisphosphonate, testosterone, and anabolic steroids are all licensed for treating osteoporosis in appropriate patients. Calcitonin, a protein hormone, cannot be given orally. It can be given by injection (and by intranasal spray-the latter is not yet approved by the Medicines Control Agency in the United Kingdom, though widely available in continental Europe). All calcitonins are 'osteoclast poisons', stopping bone loss and allowing small amounts of bone accretion by unopposed osteoblastic activity. Synthetic derivatives of human, salmon, and eel calcitonins are available — salmon calcitonins being most commonly used. Tolerance is good, serious adverse effects rare, both men and women can be treated, but the cost is high ( $£ 1000$ to $£ 2000$ a year). A pain relieving action, independent of its bone saving action, is claimed for salmon calcitonin and makes it suitable for initiating treatment in painful spinal osteoporosis, substituting a bisphosphonate later.

The bisphosphonates, derived from the pyrophosphonate molecule but with -P-C-Pcentral structure instead of $-\mathrm{P}-\mathrm{O}-\mathrm{P}-$, are resistant to pyrophosphatase. They have a high affinity for bone, where they also 'poison' osteoclasts, and stabilise microcrystals of hydroxyapatite against enzymatic dissolution. Disodium etidronate (Didronel) is the only bisphosphonate licensed for the treatment of osteoporosis in the United Kingdom and must be given intermittently. Daily treatment for two weeks is followed for the next two and a half months by daily calcium supplements, the cycle then being repeated indefinitely. This was the experimental design on which the licence for osteoporosis treatment was approved. Continuous treatment may make things worse by inhibiting the osteoclasts. Pamidronate, tiludronate, and clodronate are other successful bisphosphonates, which are not yet generally available in the United Kingdom.

Anabolic steroids are modified androgens, which build up bone and muscle, but in women will masculinise if used for three years or more, sometimes sooner. Moustache hair, deeper voice, and increased libido are unwanted effects. They and testosterone can, of course, be used in men with osteoporosis.

Sodium fluoride, used experimentally in doses of $20-80 \mathrm{mg} /$ day, restores vertebral bone density, but the larger doses do so at the expense of cortical bone, making appendicular bones more brittle. Smaller doses have been shown to reduce the spinal refracture rate, but the therapeutic interval is narrow.

Calcium should be regarded as a nutrient rather than a medicine. If the diet contains enough there is no point in giving more. But what is enough? United Kingdom guidelines have been issued by the COMA committee and are about $800 \mathrm{mg} /$ day-more during pregnancy and lactation. ${ }^{14}$ Calcium absorption from the gut is normally about $20-30 \%$ efficient, which leaves plenty of 'slack' to be taken up if other 
factors decrease dietary calcium effectiveness. A diet high in flesh protein and salt increases urinary calcium excretion, which may be why vegetarians seem to get less osteoporosis. A high phytate, high phosphate diet binds calcium and makes some of it unavailable for absorption. Vitamin D controls calcium absorption in the proximal intestine, but absorption reflects calcium concentration in the distal ileum, and so taking more in the diet will increase the total absorbed but not proportionally.

Older people are less efficient at absorbing calcium, so should take more (but often take less). The frail elderly and the disabled who seldom get out in the sun may also be marginally depleted of vitamin D.

Polypharmacy within the range of existing treatments or in combinations with candidate treatments is under investigation in many centres in the hope of promoting bone formation while stopping bone resorption. Raised professional awareness of the burden of osteoporosis has meant that the medical industry perceives a market, and this has stimulated research into new drugs.

Treatments not based on drugs may, however, have major impact on the lives of those with osteoporosis. These range from physiotherapy and exercise schedules, which will safely increase muscle strength and coordination, to car seats adapted to the shorter truncal height, to specially designed clothing to hide ugly hump backs, thus improving self confidence. Weightbearing exercises are best, but hydrotherapy is useful to get painful limbs and weak muscles going again after a spinal or hip fracture. Orthopaedic surgery has made great advances in the internal splinting of long bone fractures, though total hip arthroplasty may be the best solution for an osteoporotic fractured neck of femur.

The attitude 'Nothing can be done, dearyou'll have to learn to live with it' should now be a thing of the past.

1 Fractured neck of femur, prevention and management. London: Royal College of Surgeons, 1989: 2 .

2 Benton D. Roberts G. Effect of vitamin and mineral enton D, Roberts G. Effect of vitamin and mineral supplementation on intelligence

3 Slemenda C, Hui S L, Johnstone C C. Patterns of bone loss and physiological growing: prospects for prevention of osteoporosis by attainment of greater peak bone mass. In: Christiansen C, Overgaard K, eds. Osteoporosis Vol 2. Copenhagen, Osteopress, 1990: 948-53.

4 Law M R, Wald N J, Meade T W. Strategies for prevention of osteoporosis and hip fracture. BMF 1991; 303: 453-9.

5 Dawson-Hughes B, Dalal G E, Sadowski L, Sahyoun N, Tannenbaum S. A controlled trial of the effect of calcium Tupplementation on bone density in post of calcium women. $N$ Engl f Med 1990; 323: 878-83.

6 Wolman R L, Clark P, McNally E, Harries M, Reeve J Menstrual state and exercise as determinants of spinal Menstrual state and exercise as determinants of spinal trabecular

7 Hansen M A, Overgaard K, Riis B J, Christiansen C. Role of peak bone mass and bone loss in postmenopausal osteoporosis: 12 year study. $B M F$ 1991; 303: $961-4$.

8 Keil D P, Felson D T, Anderson J J, Wilson P W F, Moskowitz M A. Hip fracture and the use of oestrogens in post menopausal women. $N$ Engl 7 Med 1987; 317: $1169-74$.

9 Anonymous. More than hot flushes [editorial]. Lancet 1991; 338: $917-8$.

Preventing osteoporosis (letters). BMF 1991; 303: 920-2.

11 Spector T D. Use of oestrogen replacement therapy in high risk groups in the United Kingdom. $B M \mathcal{F} 1989 ; 299$ : 1434

12 Lindsay R, McKay Hart D, Krasziewski A. Prospective double blind trial of synthetic steroid (org OD 14) for preventing postmenopausal osteoporosis. BMF 1980; 281 : 1207-9.

13 Vandenbrooke J P, Witteman J G M, Valkenburg H A, et al. Non-contraceptive hormones and rheumatoid arthritis in perimenopausal women. $\mathscr{J} A M A$ 1986; 255: 1299-303.

14 Committee on medical aspects of food policy. Dietary reference values for energy and nutrients in the United Kingdom. London: HMSO, 1990: 136. (Report on Health and Social Subjects, No 41 .) 